\title{
Malignant Peripheral Nerve Sheath Tumor of the Thigh Invading the Superficial Femoral Artery, with Necrotic Lung Metastases as Presenting Symptoms
}

\author{
Patrick Mailleux ${ }^{1}$, François Buche ${ }^{2}$, Geoffrey Colin' ${ }^{1}$ \\ ${ }^{1}$ Department of Imaging, Clinique Bouge, Bouge, Belgium \\ ${ }^{2}$ Department of Vascular Surgery, Clinique St Luc, Bouge, Belgium \\ Email: p.mailleux@gmail.com
}

How to cite this paper: Mailleux, P., Buche, F. and Colin, G. (2020) Malignant Peripheral Nerve Sheath Tumor of the Thigh Invading the Superficial Femoral Artery, with Necrotic Lung Metastases as Presenting Symptoms. Advances in Lung Cancer, 9, 24-29.

https://doi.org/10.4236/alc.2020.92003

Received: April 3, 2020

Accepted: May 3, 2020

Published: May 6, 2020

Copyright $\odot 2020$ by author(s) and Scientific Research Publishing Inc. This work is licensed under the Creative Commons Attribution International License (CC BY 4.0).

http://creativecommons.org/licenses/by/4.0/

\begin{abstract}
A NF1 (neurofibromatosis 1) patient developed multiple necrotic lung metastases from a sciatic malignant peripheral nerve sheath tumor (MPNST) invading the superficial femoral artery. The first diagnosis was metastases of a non-small-cell adenocarcinoma because the right calf MPNST was not clinically noticeable ant that the chest/abdomen PET/CT did not include the region of the legs. When the MPNST was diagnosed, new histological analysis on the metastases changed the diagnosis to that of epithelioid undifferentiated sarcoma. The article deals with the sometimes-delayed diagnosis in those NF1 patients with large palpable masses and chronic pain pre-existing the malignant transformation, and discusses the difficulty of the biopsy of necrotic metastases.
\end{abstract}

\section{Keywords}

Necrotic Metastasis, Malignant Peripheral Nerve Sheath Tumor, NF1, Lung Adenocarcinoma, ${ }^{18}$ F-FDG PET/CT, Unknown Primary Tumor

\section{Introduction}

We report a case of multiple necrotic lung metastases in a NF1 patient with no proved primary tumor on cervico-thoraco-abdominal PET-CT. The first proposed diagnosis on basis of the surgical wedge lung biopsy was that of a non-small cell adenocarcinoma. Later was found deep in the thigh a large tumor invading the femoral artery, with final proposed diagnosis of malignant peripheral nerve sheath tumor (PMNST) with lung metastases. A second reading of the lung biopsy corrected the diagnosis to that of an epithelioid undifferentiated sarcoma. 
This case stresses the importance that maximal information about the clinical problem and all antecedents should be given to the anatomopathologist and it also shows that unfocused cervico-thoraco-abdominal PET-CT could miss a peripherally located primary tumor.

\section{Case Report}

A 60 year old patient presented with a deterioration of the general status and dyspnea, having lost 24 kgs during the 6 previous months. He had a 50 pack year history of smoking, severe chronic obstructive pulmonary disease, coronary artery disease and mitral valve surgery, and was a known NF1 patient since the age of 30 when a lumbar spine CT performed for right lower leg pain revealed a large plexiform lumbosacral neurofibroma [1]. He had been suffering from right lower swelling and varicose ulcers for a long time.

CT scanner of the chest and abdomen showed a right lumbo-sacral plexiform neurofibroma much enlarged since the initial diagnosis 30 years ago, multiple peripheral small hypodermic lesions and large necrotic pulmonary masses thought to be metastases (Figure 1). A ${ }^{18} \mathrm{~F}-\mathrm{FDG}$ PET/CT scanner of the neck, chest and abdomen demonstrated that the abdominal neurofibroma and the multiple subcutaneous nodules were not hypermetabolic while the pulmonary masses were only slightly hypermetabolic on the periphery (Figure 2). A hypermetabolic left adrenal nodule was also seen (Figure 2(B)).

Repeated CT-scan guided biopsies brought only necrotic tissue. So that a surgical wedge lung biopsy was performed, leading to an initial proposed diagnosis of "non-small-cell adenocarcinoma, without specific lung tumor marker, but with intense and diffuse PD-L1 membrane staining". Immunotherapy was initiated with pembrolizumab.

Three months later, the patient reported increased right lower leg pain, with a more typical arterial pattern and intermittent claudication. CT angiography of the lower limbs showed a large deep necrotic mass, of $14 \mathrm{~cm}$ in diameter, centered on the great sciatic nerve and occluding the distal superficial femoral artery (SFA) (Figure 3(A) and Figure 3(B)).

After the discovery of that large mass invading the SFA, CT images performed 2 years earlier in another hospital were obtained and reviewed (Figure 4(A) and Figure 4(B)). There was no pulmonary mass at that time. CT showed many regular poorly enhancing masses in the legs thought to be benign peripheral nerve sheath tumors (BPNST), but one of those many tumors was already avidly contrast-enhancing and had irregular borders: it was located close to the distal SFA and extended into the distal vastus medialis muscle (Figure 4(A) and Figure 4(B), stars), at exactly the same location of the necrotic tumor invading the SFA two years later (Figure $4(C)$ ). It was thus assumed that a large MPNST had developed on a pre-exiting BPNST. Supporting that assumption, the fact that a proofreading was done on the initial surgical lung biopsy sample leading to a new proposed diagnosis of epithelioid undifferentiated sarcoma without any classical tumor marker (Figure 5). 


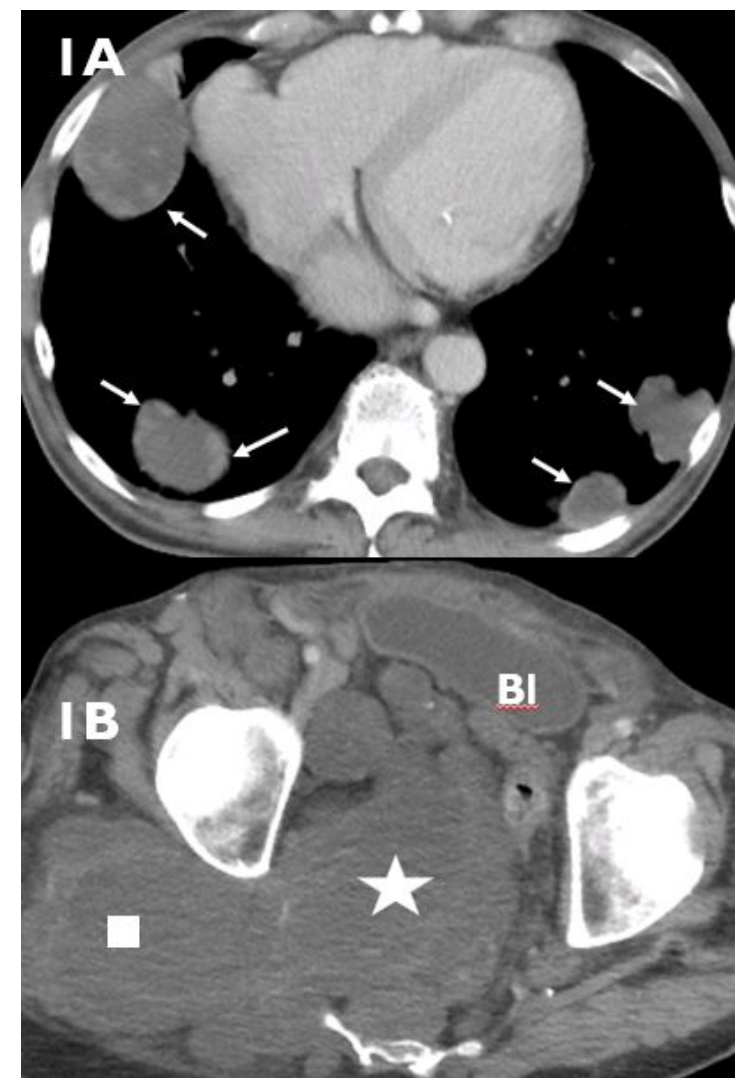

Figure 1. CT scan with intravenous contrast injection of the base of the chest (A) and of the pelvis (B). (A) Multiple necrotic masses in the lung (arrows); (B) The plexiforme neurofibroma known since 1990 has enlarged, presenting as a large tumor in the pelvis (star), extending through the right greater sciatic notch into the right deep gluteal region (square). The masses compress the bladder which is pushed towards the left side (Bl).

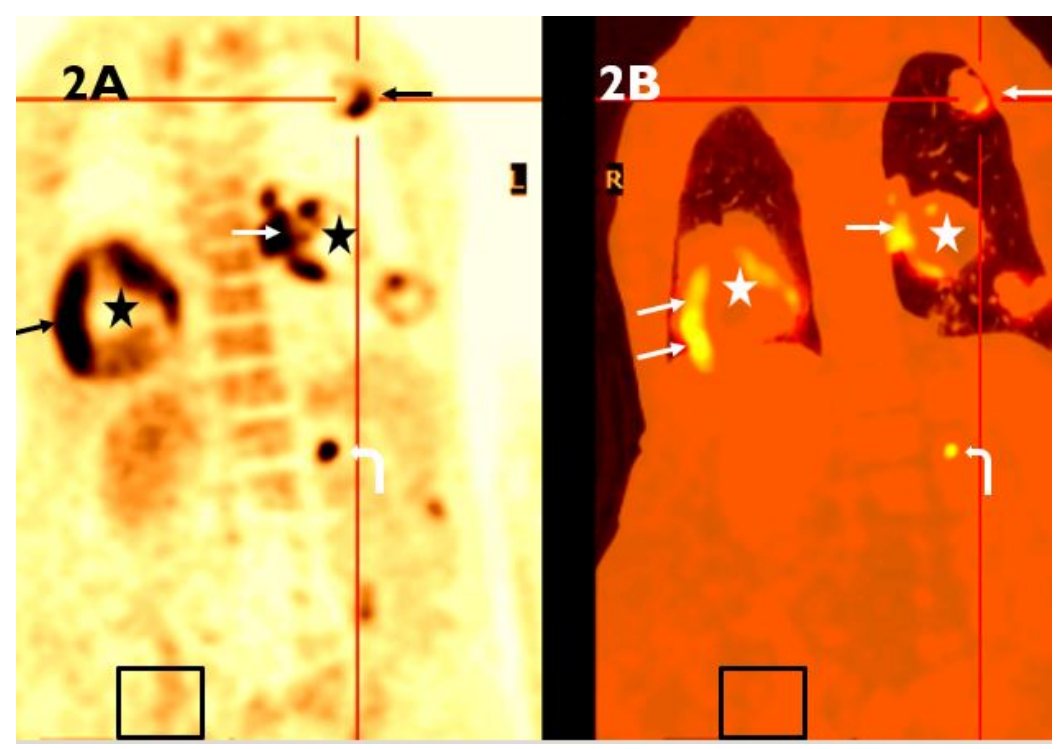

Figure 2. (A) ${ }^{18}$ F-FDG PET; (B) Fusion of PET images with CT images, coronal images. They show the necrotic lung masses (star) with partial and peripheral uptake (small arrows). No significant uptake of the right lumbosacral plexiform tumor (square). Uptake in the left adrenal area (curved arrow). 


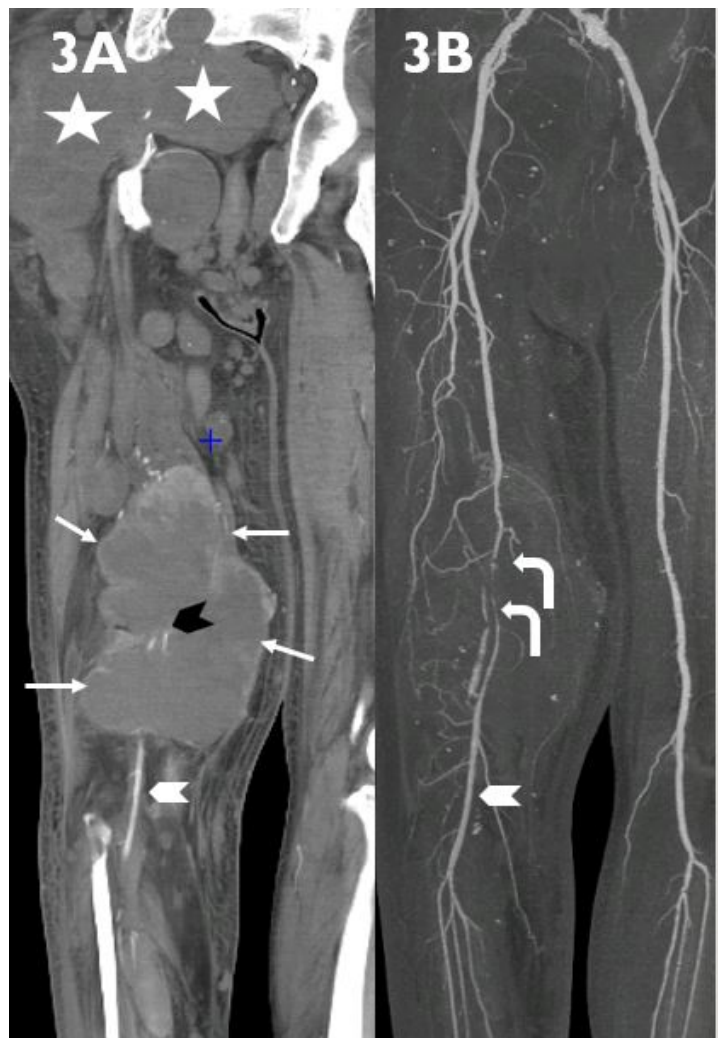

Figure 3. CT angiography of the lower limbs. (A) Coronal image showing a large necrotic tumor deep in the distal thigh (arrows), invading the distal femoral artery (black arrowhead). Distally the popliteal artery is patent (white arrowhead). The large plexiform tumor crossing the sciatic notch is also seen in the pelvis (stars); (B) MIP images showing the occlusion of the distal femoral artery at the level of the tumor (curved arrows). The popliteal artery is patent (white arrowhead).

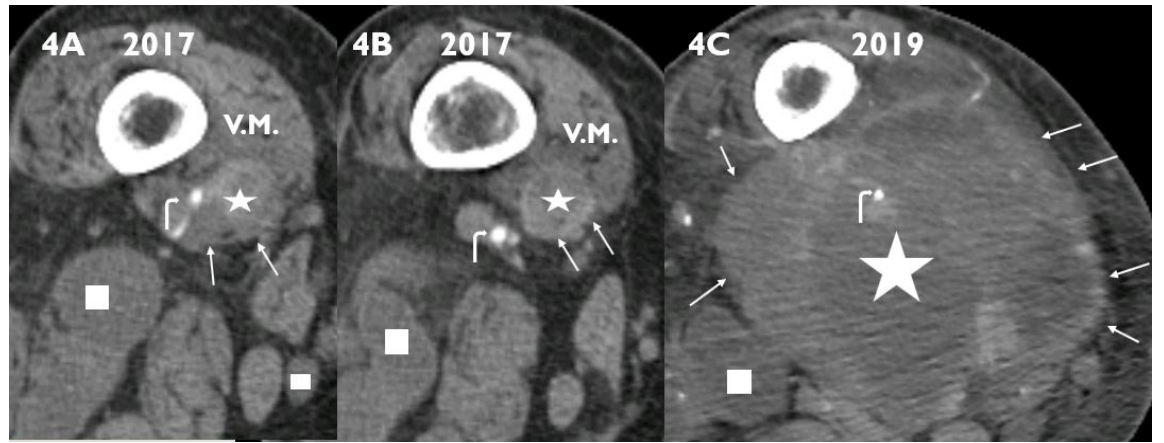

Figure 4. Axial angioCT in the thigh. (A) and (B) are from 2017, (C) from 2019, same level. On (A) and (B) poorly enhancing tumors (squares). Distal femoral artery (curved arrows) contacting an enhancing nodule (small star) with irregular borders (thin arrows) that extends into the vastus medialis muscle (V.M.). (C) Two years later, large necrotic tumor (large star) at the same location, invading artery (curved arrows) and muscles.

The new histological diagnosis did not cause treatment change because the patient was in very poor general status with cardiac insufficiency, and had showed a partial response of the lung metastases with the immunotherapy. He died two months later, 13 months after discovery of the pulmonary metastases. 


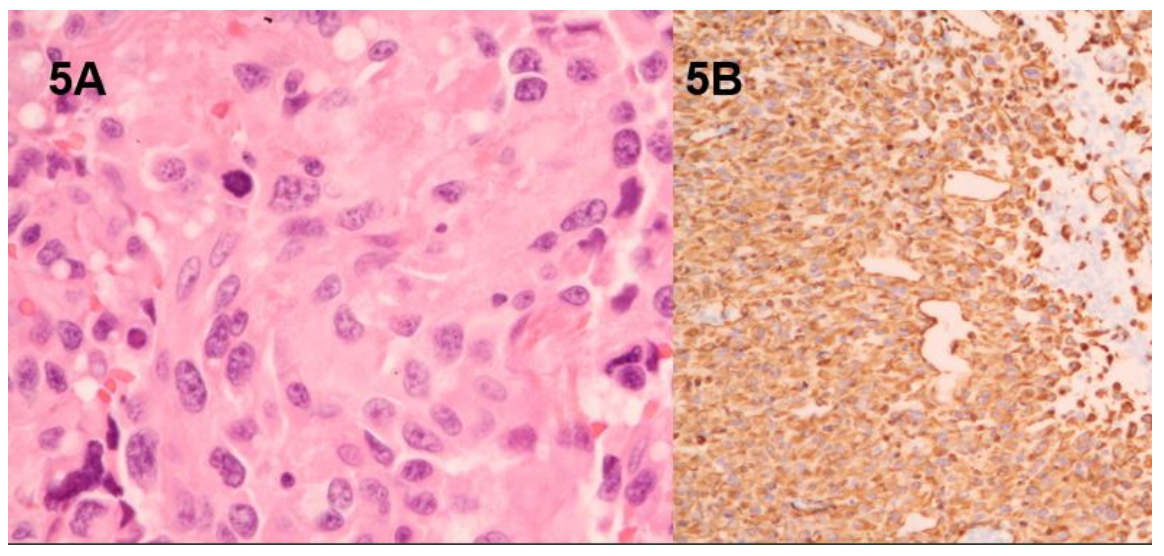

Figure 5. Lung surgical biopsy. (A) hemotoxyline/eosin coloration shows dense cellular proliferation with nuclei increased in size and one mitosis, consistent with epithelioid sarcoma; (B) Vimentine staining, which is not specific, but very sensitive for sarcomas.

\section{Discussion}

When dealing with patients with lung metastases of an unknown primary tumor, it can be difficult to obtain the diagnosis of the primary cancer. Classically, the first exam to be done, and often the more efficient, is a ${ }^{18} \mathrm{~F}$-FDG PET/CT covering head, neck, chest and abdomen with sensitivity, specificity rate and accuracy of ${ }^{18} \mathrm{~F}$-FDG PET/CT in detection of primary tumor as high as $84 \%, 78 \%$ and $82 \%$ [2].

When the primary remains unknown, image-guided biopsy is the next step. But the yield of those biopsies in necrotic tumor can be poor, sometimes with no results, or poor sample leading to incorrect diagnostic, even, as in this patient, when the biopsy was guided with the results of the ${ }^{18} \mathrm{~F}$-FDG PET/CT [3].

The patient was a known NF1 patient, with a large lumbo-sacral plexiform neurofibroma [1]. The ${ }^{18} \mathrm{~F}-\mathrm{FDG}$ PET/CT showed an increase of the size of that lesion, which was not suspect of malignancy because absence of ${ }^{18} \mathrm{~F}-\mathrm{FDG}$ uptake is rare in MPNST [4]. MPNST can appear in NF1 patients as in non-NF1 patients. A large study from the Mayo clinic [5] on a population of 4.5 million people showed an annual incidence of MPNST of 1.0 per 1000 and a lifetime risk of $8 \%$ in NF1 patients, much higher than presumed earlier. The conclusions of their study were that "patients with NF1 should be made aware of the relatively high risk of MPNST with the likely symptoms of pain and rapid growth" and that "prospective studies of ${ }^{18} \mathrm{~F}$-FDG PET/CT scanning in the highest risk patients should be considered". The median age at diagnosis in NF1 MPNST was quite younger (an average of 26 years) compared to an average of 62 years in patients with sporadic MPNST [5]. The five year survival rate was also quite lower for the NF1 MPNST patients than for the sporadic MPNST (21\% versus $42 \%)$. It could be because the NF1 patients had large palpable masses and chronic pain long before the malignant transformation. Or, as in our patient, because of the long standing right lower varicose ulcers and swelling.

In a large series of 65 patients with MPNST [6], 18 patients had distant metastases at the time of presentation or developed them in the course of follow-up, 
only two patients had metastases without lung involvement. Without metastases the overall survival was 126 months, while it dropped to 36 months with metastases. Those authors claim that amputation should be the last resort because it gives no benefit in survival and that functional reconstruction should be performed early by tendon transfer and not by nerve transplantation, in order to improve quality of life.

\section{Conclusion}

In NF1 patients with metastatic malignancy and unknown primary cancer, the possibility of MPNST should be kept in mind because it is less infrequent than earlier thought. In those specific cases, total body ${ }^{18} \mathrm{~F}$-FDG extended to the lower legs could be asked and the pathologists having to examine the performed biopsies must be informed of the likeliness of a sarcomatous tumor of the nerve sheath.

\section{Conflicts of Interest}

The authors declare no conflicts of interest regarding the publication of this paper.

\section{References}

[1] Coulier, B. and Mailleux, P. (1990) Pelvic Plexiform Neurofibroma: A Typical CT Pattern. Journal Belge de Radiologie, 73, 97-101.

[2] Cengiz, A., Göksel, S. and Yürekli, Y. (2018) Diagnostic Value of ${ }^{18}$ F-FDG PET/CT in Patients with Carcinoma of Unknown Primary. Molecular Imaging and Radionuclide Therapy, 27, 126-132. https://doi.org/10.4274/mirt.64426

[3] Chockalingam, A. and Hong, K. (2015) Transthoracic Needle Aspiration: The Past, Present and Future. Journal of Thoracic Disease, 7, S292-S299.

[4] Ferner, R.E., Lucas, J.D., O’Doherty, M.J., Hughes, R.A., Smith, M.A., Cronin, B.F. and Bingham, J. (2000) Evaluation of ${ }^{18}$ Fluorodeoxyglucose Positron Emission Tomography $\left({ }^{18} \mathrm{FDG}\right.$ PET) in the Detection of Malignant Peripheral Nerve Sheath Tumors Arising from within Plexiform Tumors in Neurofibromatosis. Journal of Neurology, Neurosurgery \& Psychiatry, 68, 353-357.

https://doi.org/10.1136/jnnp.68.3.353

[5] Stucky, C.H., Johnson, K.N., Gray, R.J., et al. (2012) Malignant Peripheral Nerve Sheath Tumors (MPNST): The Mayo Clinic Experience. Annals of Surgical Oncology, 19, 878-885. https://doi.org/10.1245/s10434-011-1978-7

[6] Goertz, O., Langer, S., Uthoff, D., Ring, A., Stricker, I., Tannapfel, A. and Stenau, H. (2014) Diagnosis, Treatment and Survival of 65 Patients with Malignant Peripheral Nerve Sheath Tumors. Anticancer Research, 34, 777-783. 